Adenosine $3^{\prime}, 5^{\prime}$-monophosphate developmental biochemistry fetus

glucagon

liver norepinephrine

phosphoenolpyruvate carboxykinase prednisolone

tyrosine transaminase

\title{
Enzyme Response to Prednisolone and Dibutyryl Adenosine 3', 5'-Monophosphate in Human Fetal Liver
}

\author{
Lorne Kirby and Peter Hahn ${ }^{[16]}$ \\ Departments of Pacdiatrics and Obstetrics and Gynaecology, University of British Columbia, Vancouver, British Columbia, Canada
}

Extract

In human fetal liver cultures addition of glucagon or dibutyryl adenosine $3^{\prime}, 5^{\prime}$-monophosphate (dibutyryl cyclic AMP) plus prednisolone to the medium for $24 \mathrm{hr}$ evokes an increase in tyrosine $\alpha$-ketoglutarate transaminase (TTA) activity which is much greater than the sum of the rises due to glucagon, dibutyryl cyclic AMP, or prednisolone alone. This effect is time ( $24 \mathrm{hr}$ of incubation) and age (the largest effect in the oldest fetus) dependent. This synergistic effect is not seen with phosphoenolpyruvate carboxykinase (PEPK). Glucagon alone or norepinephrine alone has only a very dubious effect on TTA but both hormones raise PEPK activity by about $60 \%$ in culture.

Alanine and aspartate transaminases are not affected by any of the inducers tested, but both increase in activity during culturing.

\section{Speculation}

Theoretically, it is possible that injections of steroids to pregnant mothers together with higher than normal levels of dibutyryl cyclic AMP in fetal liver can cause large increases in fetal hepatic tyrosine transaminase. The metabolic consequences of such a rise for the fetus are completely unknown. It is not even known whether such a rise would be permanent or transient. In view of our ignorance, we should be hesitant about treating pregnant women with steroid drugs.

\section{Introduction}

It has been shown in previous work [3] that addition of dibutyryl cyclic AMP to the culture medium of human fetal liver in vitro causes a rise in the activities of TTA (EC. 2.6.1.5) and PEPK (EC. 4.1.1.32). In the same paper we also reported the case of one fetus, the mother of which had been receiving prednisolone for 8 days before abortion. The PEPK activity in the liver of that fetus was more than 10 times greater than in any other fetal liver examined. It was hence decided to investigate the effect of prednisolone in vitro, either added alone or in combination with other agents. The effect of steroids on TTA in human fetal liver in culture has been examined by Räihä et al. [5], who reported an effect in a 28-week-old fetus but not in younger ones. In rat fetuses, on the other hand, steroids do have an inductive effect [6].

\section{Materials and Methods}

Fetuses aged 9-21 weeks were obtained from legal abortions within $5 \mathrm{~min}$ after hysterotomy. Liver cultures were established as described previously [3], but the 
culture medium was Eagle's minimum essential medium in Earle's balanced salt solution. The gas phase was $5 \% \mathrm{CO}_{2}$ plus $95 \% 0_{2}$.

The activities of TTA and PEPK were determined as described in a previous paper [3]. Alanine transaminase (ALT, EC. 2.6.1.2) and aspartate transaminase (AST, EG. 2.6.1.1) were determined according to the method of Bergmeyer and Bernt [2].

Inducing agents were added to the incubation medium to give the following final molar concentrations: dibutyryl cyclic AMP $9 \times 10^{-4} \mathrm{M}$, norepinephrine (NE) $1 \times 10^{-4} \mathrm{M}$, glucagon $1 \times 10^{-4} \mathrm{M}$, insulin $5 \times$ $10^{-5} \mathrm{M}$, and prednisolone $\mathrm{l} \times 10^{-5} \mathrm{M}$.

Chemicals were obtained from Sigma Chemical Co. [9] and sodium $\left({ }^{14} \mathrm{C}\right)$ bicarbonate was purchased from Amersham/Searle [10]. Radioactivity was counted in a Picker-Nuclear Chicago [11] scintillation counter.

\section{Results}

\section{Alanine and Aspartate Transaminase}

Because both enzymes have been implicated in gluconeogenesis and rise in activity after birth in rat liver [8], fetal organs were examined for their activities. Soluble ALT activity was low in the liver, heart, and kidney and of the same order of magnitude as in rat fetal liver [8]; there were very slight changes between weeks 9 and 21. Soluble AST was much higher in these organs and again was of the same order of magnitude as described for the rat [8]. No developmental changes were noted.

The TTA activity in fetal liver increases during culturing. The same was observed for both AST and ALT. After $24 \mathrm{hr}$ in culture the rise in activity for ALT was between $150 \%$ and $500 \%$ and that for AST about $50 \%$. In the oldest fetus the rise was smallest (Fig. 1). None of the agents tested, when added for 24 hr of culturing, had any significant effect on AST or ALT.

\section{Tyrosine Transaminase}

We have shown that addition of dibutyryl cyclic AMP to the culture medium causes a rise of about $50 \%$ in liver TTA activity [3]. This was confirmed in the present series of experiments. Addition of prednisolone had about the same effect as dibutyryl cyclic AMP. However, if prednisolone and dibutyryl cyclic AMP were added together, TTA activity was increased up to $700 \%$ depending on the age of the fetus (Fig. 2). In order to obtain the full effect, it was necessary that both substances be present for $24 \mathrm{hr}$ (Fig. 3).

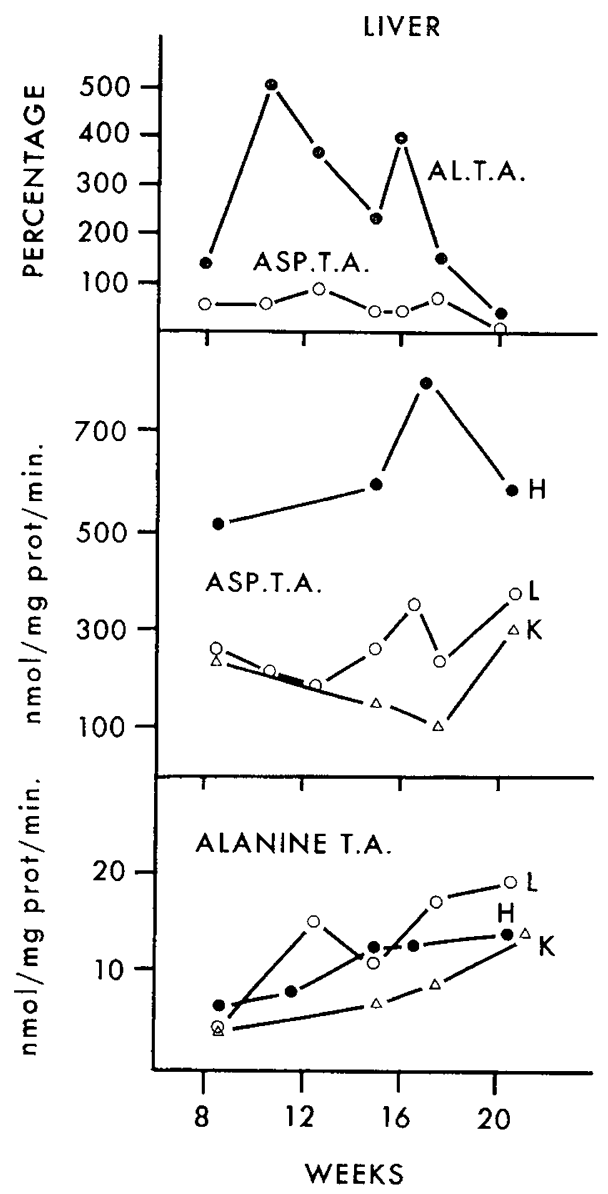

Fig. 1. Changes in cytoplasmic alanine transaminase (AL.T.A., alanine $T . A$.$) , bottom, and aspartate transaminase (ASP.T.A.),$ middle, in human fetal organs during development. Top: percentage change relative to control subjects in the activitics of both enzymes in fetal liver after $24 \mathrm{hr}$ of culturing. $L$ : liver; $H$ : heart; $K$ : kidney. The increase of alanine and aspartate transaminase due to culturing is significant for $P<0.01$, if all fetuses are considered. Activitics were determined in the $100,000 \times g$ supernatant.

Addition of glucagon to liver cultures had no, or only a slight, effect. If added together with prednisolone, a $600 \%$ rise in TTA activity was noted in the oldest fetus. In younger fetuses, however, no such effect was seen.

In contrast to glucagon, NE addition together with prednisolone increased TTA activity by only $100 \%$ in the oldest fetus. It was without effect in younger age groups (not shown).

Addition of insulin to human fetal liver cultures for $24 \mathrm{hr}$ had opposite effects depending on conceptional age. In fetuses aged less than 14 weeks, a significant increase in TTA activity was observed. In older ones there was a significant clecrease (Fig. 4). Insulin had no 
effect on the rise in TTA activity brought about by dibutyryl cyclic AMP.

\section{Phosphoenolpyruvate Carboxykinase}

Prednisolone increased PEPK activity in fetal liver cultures by an average of $20 \%$ at all ages. If dibutyryl cyclic AMP were added together with prednisolone, an additive effect was observed only in the oldest fetus.

Addition of NE to the culture medium increased activity by an average of $60 \%(P<0.01)$, and adding prednisolone to NE had no further effect (Table I).

The effect of glucagon was equivocal. In three very young fetuses, PEPK activity was raised by $56 \%$. Only very slight effects were noted in older fetuses and a rise of $85 \%$ was seen in the oldest specimen. Addition of prednisolone together with glucagon in two fetuses (17 and 21 weeks) caused PEPK activity to rise by over $120 \%$, the effect being more than additive.

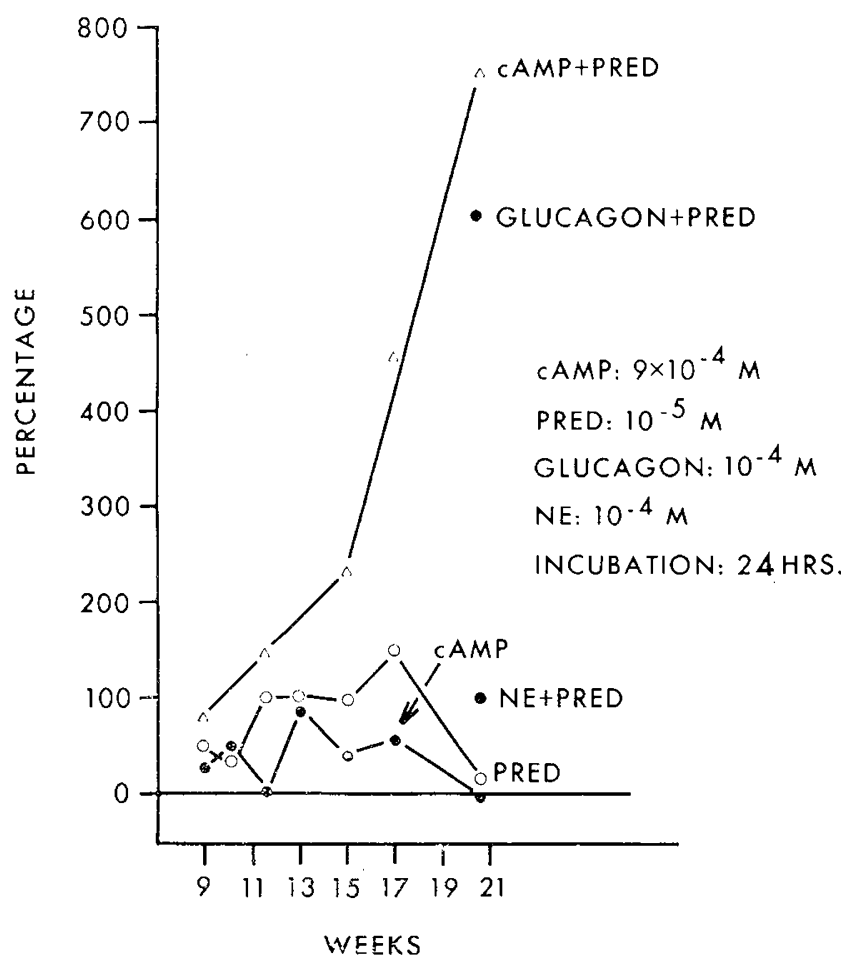

Fig. 2. The effect on liver cultures from fetuses aged 9-21 wecks of glucagon, prednisolone (PRED), dibutyryl adenosine $3^{\prime}, 5^{\prime}$ monophosphate (CAMP) and norepinephrine $(N E)$, and of prednisolone combined with the other inducers on tyrosine transaminase in human fetal liver cultured in the presence of these substances for $24 \mathrm{hr}$. Ordinate: percentage increase over control values. The coefficient of correlation for age against tyrosine transaminase activity after dibutyryl adenosine $3^{\prime}, 5^{\prime}$-monophosphate plus prednisolone is $0.925 \pm 0.44, P<0.02$.

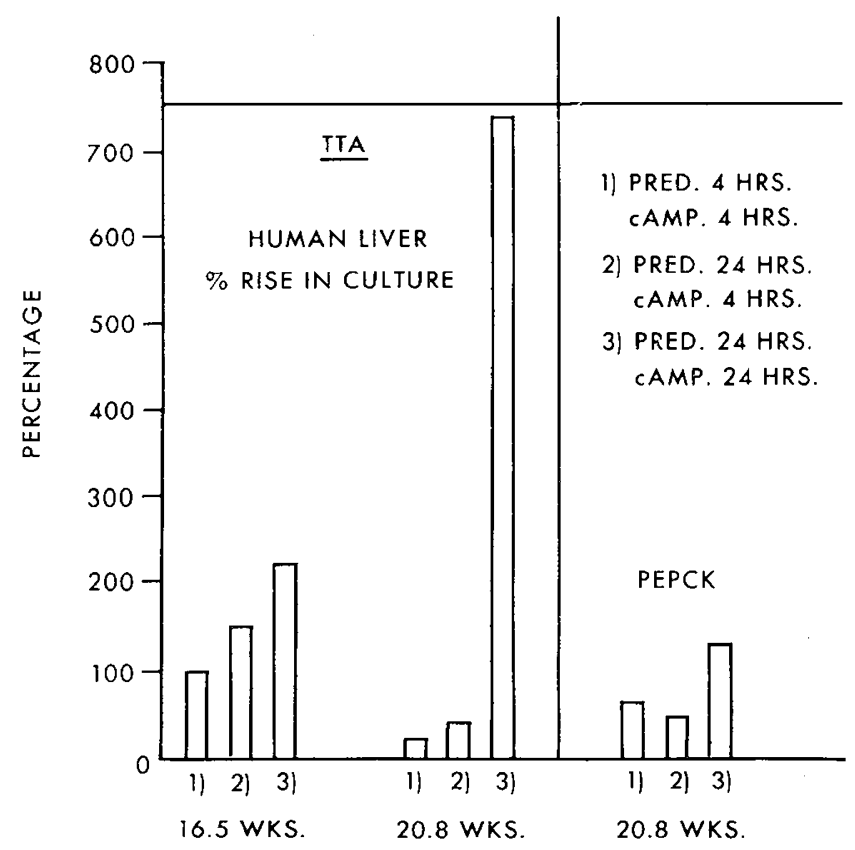

Fig. 3. The effect of timc of incubation with prednisolone (PRED.) and dibutyryl adenosine $3^{\prime}, 5^{\prime}$-monophosphate (cAMP.) on tyrosine transaminase $(T T A)$ and phosphoenolpyruvate carboxykinase (PEPCK) activities in human fetal liver. Abscissa: age of fetus.

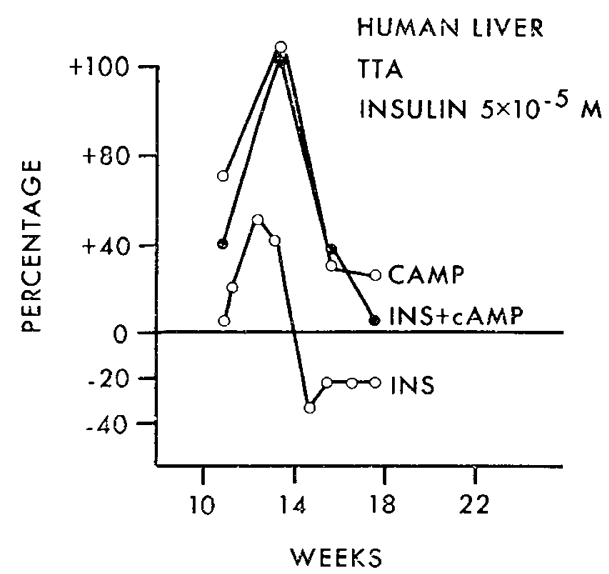

Fig. 4. The effect of insulin and dibutyryl adenosine $3^{\prime}, 5^{\prime}$-monophosphate $(C A M P, C A M P)$ on tyrosine transaminase (TTA) activity of human fetal liver cultures. Abscissa: conceptional age; ordinate: percentage change from control value. The increase in activity for the four youngest fetal livers with insulin (INS) and the decrease for the foux oldest oncs are significant for $P<0.01$.

\section{Discussion}

A large drawback of work with human fetuses is the small number of samples available. Even though all points for any one fetus were always obtained from duplicate or even triplicate determinations, and al- 
Table $I$. Effect of different agents on phosphoenolpyruvate carboxykinase activity in human fetal liver ${ }^{1}$

\begin{tabular}{cccccccc}
\hline Age & Cyclic AMP & Cyclic AMP + PR & Glucagon & GL + PR & NE & NE + PR & PR \\
\hline $11-13(3)$ & & & $56 \pm 12$ & & $10 \pm 3$ & $20 \pm 3$ \\
$14-17(6)$ & $40(1)$ & $18(1)$ & $5.56 \pm 5$ & $140(1)$ & $75 \pm 6(3)$ & $50(1)$ & $20 \pm 4$ \\
$21 .(1)$ & 72 & 130 & 85 & 120 & 130 & 130 & 24 \\
\hline
\end{tabular}

1 Values are percentage increase over control values. Figures in parenthesis refer to number of fetuses examined. Conditions as in Figure 2. All agents were present for the whole 24-hr period of culturing. Cyclic AMP: adenosine $3^{\prime}, 5^{\prime}$-monophosphate; PR: prednisolone; GL: glucagon; NE: norepinephrine.

though statistical analysis was applied whenever possible, there is no doubt that over the years we shall have to repeat these experiments as more fetuses become available. Nevertheless, in agreement with previous work, we have shown that both PEPK and TTA increase in activity in response to dibutyryl cyclic AMP. In this paper the effect of prednisolone was examined, since in vivo administration of this substance had elevated fetal liver PEPK in one pregnant woman. It was found that in vitro prednisolone alone caused a rise in TTA by about $50 \%$ and in PEPK by about $20 \%$. In the case of TTA, however, addition of prednisolone together with dibutyryl cyclic AMP or glucagon caused a rise in activity that was up to more than $600 \%$ of the control value. This was not found for PEPK. One might suggest that the difference between the response of the two enzymes is due to their different turnover rates. In rats the half-life for TTA is approximately 3 hr $[1,7]$, whereas that for PEPK is about $6 \mathrm{hr}[1,7]$; it is possible that a similar difference exists in man. This is being examined.

It would be tempting to speculate that prednisolone addition leads to the appearance of the glucagon receptor of liver adenylcyclase and that hence a more than additive effect on TTA activity was found in the oldest fetus. This hypothesis, however, must be rejected because the same phenomenon was observed when dibutyryl cyclic AMP and prednisolone were combined, even in younger fetuses. Because the effect is much more apparent after $24 \mathrm{hr}$ than after $4 \mathrm{hr}$, it is probably due to enzyme induction and not activation. It is also probable that prednisolone and dibutyryl cyclic AMP do not act at the same site; presumably the former acts on transcription and the latter on translation as has been suggested for rat liver [7]. This will have to be examined. Neither glucagon nor NE alone had any effect on TTA activity in the absence of prednisolone. Inasmuch as dibutyryl cyclic AMP was effective alone, it might be proposed that the receptors of adenylcyclase for these two hormones are not yet present. However, because NE but not glucagon always caused a rise in
PEPK activity, one must assume that at least the NE receptor is developed. Recent data [4] show that liver adenylcyclase from human fetal liver responds to both $\mathrm{NE}$ and glucagon. Our preliminary results indicate that this is true even after $24 \mathrm{hr}$ of culturing.

At this time we have no explanation for the change with age in the effect of insulin on TTA activity, nor do we care to put forward any speculation.

It may be pointed out that culturing alone leads to rise in PEPK, ALT, and AST activities, i.e. gluconeogenic enzymes, of which, however, only PEPK responds to the agents tested.

\section{Summary}

Human and rat fetal livers were cultured in vitro in Eagle's minimum essential medium. Culturing for 24 hr caused a rise in cytoplasmic alanine and aspartate transaminase, but addition of glucagon $\left(1 \times 10^{-4} \mathrm{M}\right)$, norepinephrine $\left(1 \times 10^{-4} \mathrm{M}\right)$, or dibutyryl cyclic AMP $\left(9 \times 10^{-4} \mathrm{M}\right)$ had no effect.

Tyrosine $\alpha$-ketoglutarate transaminase activity was increased by up to $700 \%$ in the presence of dibutyryl cyclic AMP and prednisolone $\left(1 \times 10^{-5} \mathrm{M}\right)$. It was necessary that both agents be added together for 24 hr. A positive correlation between fetal age and the increase due to those two agents was found. Phosphoenolpyruvate carboxykinase activity was increased by both NE and by combining prednisolone with glucagon. These data indicate that in human fetuses aged 12-20 weeks, hormonal effects on TTA and PEPK can be demonstrated in the liver.

\section{References and Notes}

1. Barnett, C. A., And Wicks, W. D.: Regulation of phosphoenolpyruvate carboxykinase and tyrosine transaminase in hepatoma cell cultures. J. Biol. Chem., 246: 7201 (1971).

2. Bergmeyer, H. U., and Bernt, E.: In: H. U. Bergmeyer: Methods of Enzymatic Analysis, pp. 837, 846 (Academic Press, New York, 1965).

3. Kiriby, L., AND Hahn, P.: Enzyme induction in human fetal liver. Pediat. Res., 7: 75 (1973). 
4. Menon, K. M. J., Giese, S., And Jaffe, R. B.: Hormone and fluoride sensitive adenylate cyclases in human fetal tissue. Biochim. Biophys. Acta, 304: 203 (1973).

5. Rärhӥ, N. C. R., Schwartz, A. L., ANd Lindroos, M. C.: Induction of tyrosine-x-ketoglutarate transaminase in fetal rat and fetal human liver in organ culture. Pediat. Res., 5: 70 (1971).

6. Wicks, W. D.: Differential effects of glucocorticoids and adenosine $3^{\prime}, 5^{\prime}$-monophosphate on hepatic enzyme synthesis. J. Biol. Chem., 246: 217 (197I).

7. WICKs, W. D.: Regulation of hepatic enzyme synthesis by cyclic AMP. Ann. N. Y. Acad. Sci., 185: 152 (1971).

8. Yeunc, D., AND Oliver, I. T.: Gluconeogenesis from amino acids in neonatal rat liver. Biochem. J., 103: 744 (1967).

9. St. Louis, Mo.
10. Arlington Heights, Ill.

11. Des Plaines, Ill.

12. We wish to express our appreciation to Dr. Betty Poland and her staff for their assistance in obtaining fetal tissue.

13. Dr. L. Kirby was supported by Vancouver Foundation Grant no. 65-8956.

14. Dr. P. Hahn is a Medical Research Council (Canada) Associate.

15. This research was supported by Medical Research Council (Canada) Grant no. 68-3713.

16. Requests for reprints should be addressed to: PETER Hahn, M.D., Faculty of Medicine, Department of Obstetrics and Gynecology, Vancouver General Hospital, The University of British Columbia, Vancouver 8, British Columbia, Canada.

17. Accepted for publication September 12, 1973. 